\title{
Example of industrial valorisation of derivative products of Castor oil
}

\author{
Patrick BORG \\ Guillaume LÊ \\ Stéphanie LEBRUN \\ Bernard PÉES \\ Pôle Synthèse des Matériaux, \\ Groupe IPOM-LSM 6, \\ 27470 Serquigny, France \\ $<$ bernard.pees@arkemagroup.com>
}

\begin{abstract}
Known since antiquity, Castor Oil has been first used in medicine. Now, even if it remains present in small quantities as an excipient in many pharmaceutical specialties, it finds a lot of applications in cosmetics, industrial applications and chemical industry. Castor Oil specificity comes from its high content of ricinoleic acid (up to 85\%) that combines a double bond and an hydroxyl function in the heart of a 18 carbons linear chain. This particular structure is the key of an unique chemistry developed by ARKEMA that gives by thermal cracking a wide range of compounds with either 7 or 11 carbon atoms. A whole range of innovative chemistries and end use products are generated from these base reaction products. They are used in every-day life, to improve our comfort and safety but also in very specific applications with very high technical requirements. Synthesized from undecylenic acid, 11 -amino-undecanoic acid, 100\% based on renewable resources, is the precursor to biobased polymers combining high performance and sustainability: Rilsan ${ }^{\circledR}$, Rilsan Fine Powder ${ }^{\circledR}$, Pebax Rnew ${ }^{\circledR}$.
\end{abstract}

Key words: Castor Oil, ricinoleic acid, oenanthol, C7 derivatives, undecylenic acid, $\mathrm{C} 11$ derivatives
Nature owes a lot to Castor Oil... starting with beavers. Indeed, very long-time hunted for their fur and Castoreum exploitation, they owe their survival to the replacement of Castoreum by Castor Oil for its medicinal "virtues" and many applications in perfumery. From the latin word castor, Castor Oil keeps only the name in memory of its applications, but it has absolutely nothing in common chemically. Regarding the name ricinus, it is in reference to the similarity between Castor Beans and ticks that the Swedish naturalist Carolus Linnaeus was giving scientific name to ricinus communis plants over 200 years ago.

Despite all these links with the animal world, Castor Oil is a product of plant origin. Castor Oil is obtained from Castor Beans Plants mainly cultivated in sub-tropical regions (India, Brasil, China) that fructifies all along the year (several harvests in a year) and is drought resistant. It is a robust culture (very few pesticide), Castor Beans Plants can be grown on poor soil in semi arid areas, do not compete with local food crops nor contribute to deforestation. To ensure only ripe seeds collection harvesting is done manually (mechanization is very limited).

Castor Oil is obtained by crushing Castor Beans seeds that come from Castor Plants where they are located in Spiny Seed Pod. It is obtained either by cold-pressing or by solventextraction of seeds containing 40 to $60 \%$ of oil. Ricin is a toxin present in the seed and plant but due to its high hydrophilic behavior it is not soluble in oil. Then it concentrates in the Oil Cake that is heat-treated $\left(125^{\circ} \mathrm{C} /\right.$ $25 \mathrm{~min}$ ) to destroy the toxin and valorized as organic fertilizer for biological agriculture or used as a feedstock for livestock. In conclusion, Ricin is not found in oil.

Castor Oil is not used for human feeding. Known since antiquity, castor oil has been used in medicine as a purgative laxative stimulant. Now, it remains present in small quantities as an excipient in many pharmaceutical specialties. In cosmetics, it is used as base of beauty oils for hands, body and especially hairs. It is used under the form of shampoo or hair masks, but also as a plasticizer in nail polish, lipsticks, creams and lotions, or as color fixative. Castor oil has also industrial applications because of its polyol ester's structure which acts as a lubricant for engines operating at very high speed. It is used in the chemical industry in the polyurethane sector, as a component of polyglycerol polyricinoleate (emulsifier for food industry), in the manufacture of lacquers, varnishes, polishes and wax substitutes. It also finds applications under a dehydrated (DCO), hydrogenated (HCO) or sulphated (Turkey Red Oil) form.

The originality of Castor Oil relies in its chemical nature. The specificities come from: - The high content (up to $85 \%$ ) of a particular fatty acid: the ricinoleic acid.

- The unique structure of ricinoleic acid: at the difference of others conventionnal fatty acids from vegetable oils, it combines a double bond and an additionnal hydroxyl functionnality inside its linear chain comprising 18 carbon atoms.

After alcoholysis reaction, a mixture of glycerol and fatty acids esters is obtained with a high percentage of ricinoleic acid ester (almost 85\%).

Glycerol is a multifunctionnal organic compound, soluble in water, and is widely used in many application fields: wetting and lubrificating agent in handcreams, suntan lotions and soaps hair care, anti-freezing, biobased raw materials for chemical intermediates such as acrolein, propylene glycol, epichlorhydrin...

The behaviour of Ricinoleic acid ester when heated to high temperature has intrigued chemists since 1845. Since that time Castor Oil chemistry has evolved significantly. The commonly way is to crack under alkaline conditions (caustic pyrolysis) leading to capryl alcohol (2-octanol) and sebacic acid (C10 a, w-diacid). The process in use at the Arkema Marseilles plant is in fact unique in the world. After alcoholysis reaction with methanol, methyl ester of ricinoleic acid is cleaved by steam cracking (pyrolysis at elevated temperature) into two parts giving first a 7 carbon atoms compound (heptanal) and a 11 carbon atoms compound (methyl undecylenate) (figure 1).

A whole range of innovative chemistries and end use products are generated from these base reaction products. These products are used in every-day life, to improve 


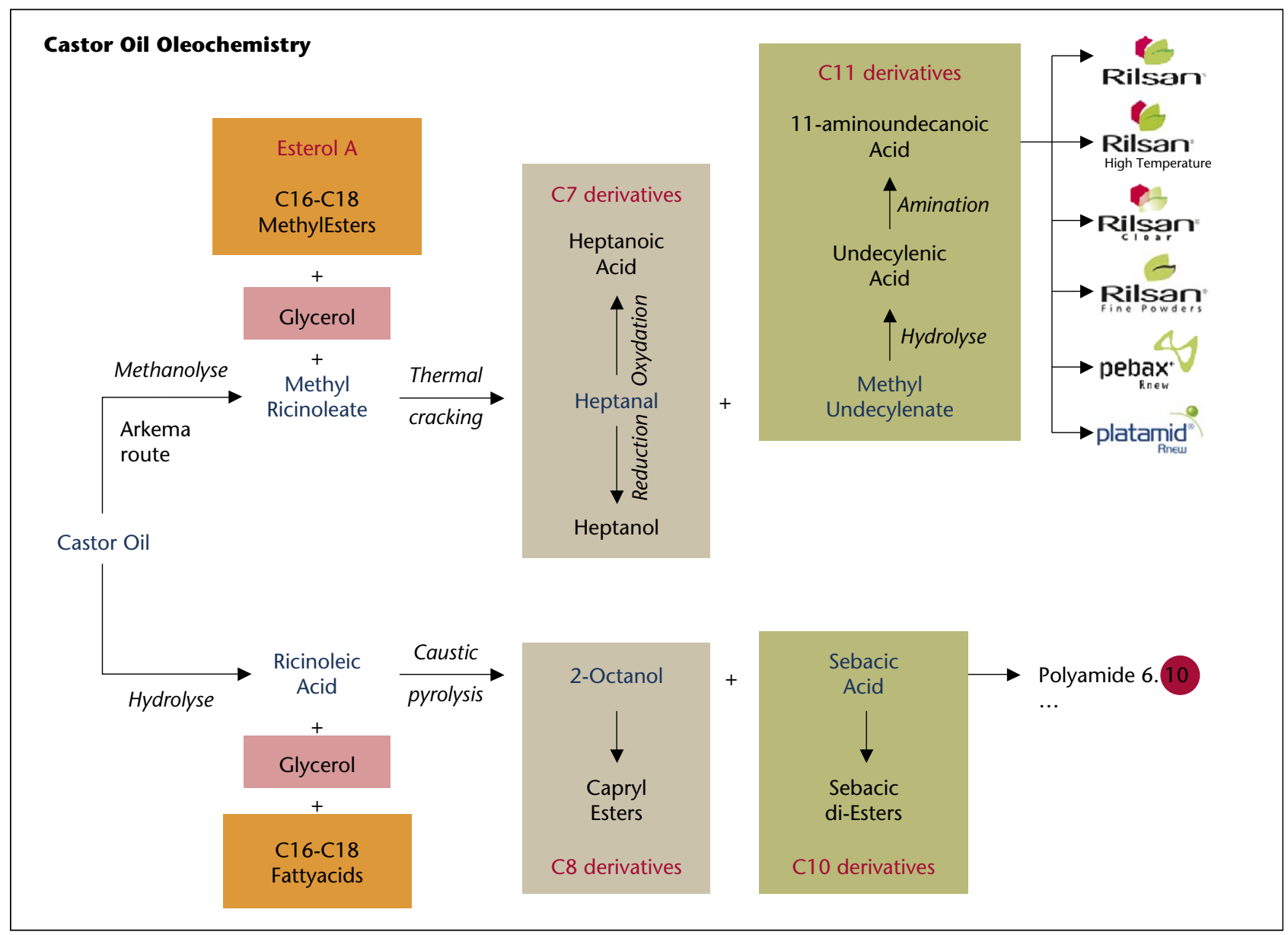

Figure 1. Castor Oil Oleochemistry.

our comfort and safety but also in very specific applications with very high technical requirements.

\section{When $18=10+8 \ldots$}

As described before, the most practiced way is to carry out the cracking reaction under alkaline conditions (caustic pyrolysis) leading to different final products: capryl alcohol (2-octanol) and sebacic acid (C10 a, w-diacid).

Sebacic acid is a linear saturated 10-carbon dicarboxylic acid. The major part of world production occurs in China (over 20,000 metric tonnes of exportation, over $90 \%$ of global trade). In the industrial setting, sebacic acid can be used as such or as an intermediate in lubricants, hydraulic fluids, cosmetics, candles, aromatics, antiseptics and painting materials. But Sebacic acid can be used for partially bio resourced polymers: for example, by coupling with hexamethylene diisocyanate: nylon-6,10. Furthermore, sebacic acid esters are used as plasticizers for different polymers and synthetic rubbers (dibutyl sebacate DBS) and in the manufacture of dioctyl sebacate (jet lubricant and lubricant in aircooled combustion motors).

The 2-octanol (capryl alcohol) is mainly used as a raw material to produce caproic acid intermediates in butter, rum, coconut and fruit flavors. In cosmetics, it is an intermediate for the preparation of caprylic/capric triglyceride (CCT): a mixed triester of caprylic and capric acids possessing excellent oxidation stability (almost indefinite shelf life). CCT is an emollient, excellent as a superfatting oil in soapmaking and improves spreading of skincare formulas. Capryl alcohol can be used as a possible alternate for 2-ethylhexanol or isooctyl alcohol in the preparation of diesters plasticizers: dicapryl esters such as dioctyl phthalate (DOP) or dioctyl adipate (DOA). The 2-octanol is used as a solvent, but it is also employed as a frother in the mineral froth flotation of silica from iron ore.

\section{When $18=11+7$}

Arkema has developed a whole range of compounds with 11 and 7 carbon atoms derived from a chemistry based on the thermal cracking of ricinoleic derivatives.

\section{Esterol A: "the remaining C18 part"}

It corresponds to the not breaking part after steam cracking. This product is made up of saturated and unsaturated natural fatty acid methyl esters like stearic, oleic or linoleic acid. They consist of C16 and C18 linear chains, identical to those contained in the common vegetable oils, and are valorized for their value in the oil $\&$ fat markets. Esterol $A$ is mainly used as machining oils due to its capacity to fix sulphur and a good ability to prevent scuffing or scoring of metal parts due to good lubricating properties (oilness, anti-wear properties). It finds a lot of applications in a large range of domains:

- Metal working fluids/Cutting oils (lubricity for anti-wear additive when sulfurized).

- Fat liquors for leather treatment.

- Concrete mold release agents (biodegradable, fluid oil, easy to emulsify).

- Grease and lubrication formulations.

- Anti-foam agents. 


\section{"The C7 cut" and its derivatives... from perfumes to technical products}

Heptanal, a molecule with 7 aligned carbon atoms, is appreciated for the olfactory qualities of certain of its derivatives. In its natural state it was discovered in wine by oenologists who called it Oenanthol. Other, more technical qualities were discovered later, including its lubricant properties thanks to the low freezing point of this chain. Heptanal is a synthesis intermediate for the fragrance and aroma industry. Its natural odor is categorized as fresh, green, citrus and is used in aldehydic and floral compositions. Synthetic jasmine or jasminaldehyde (ACA: a-n-amyl-cinnamaldehyde) is a perfume aroma obtained from $\mathrm{C} 7$ aldehyde. This aroma is detectable in many washing powders, soaps, candies and other jasmine perfumed products. Another perfume aroma made up from heptanal is Folione (methyl-heptyne carboxylate). With its green, violet, vegetable and hay like odor, Folione is used in fragrances that require a hay or straw-like quality.

Heptanal is transformed into 2 new compounds either by oxidation or by reduction leading respectively to heptanoic acid and heptanol.

Heptanoic acid has a large variety of applications. It can be valorized in the form of esters as civil and military aircraft jet engines lubricant, car motor oil and refrigerant lubricants. Compared to $\mathrm{C} 6 / \mathrm{C} 8 / \mathrm{C} 10$ analogues, linear saturated C7 acid esters based on trimethylolpropane, neopentylglycol, pentaerythritol present a better compromise in terms of low viscosity at low temperature and low volatility at high temperature due to their lower pour points and higher viscosity indexes. In addition to lubricant behavior, C7 salts have high efficiency as corrosion inhibitor for water based hydraulic fluids for automotive, additives in paints, cutting oils with C7 monoethanolamides and metalworking fluids. Heptanoic acid is a chemical intermediate for many application fields as:

- pharmaceuticals and cosmetics (emollient agent/surfactant in personal care as propylene glycol diheptanoate, stearyl heptanoate and in pharmaceutical gel as neopentylglycol diheptanoate);

- aromas and perfumes (natural, green grassy and fruity ester like odor in banana flavors and in coffee, dairy products, passion fruit...);

- plasticizers for polymers.

Heptanol is used in the fragrance and aroma industry (trace quantities are used in perfumes to provide a peach-apricot aroma) or as a synthesis intermediate (g-decalactone as coconut, vanilla flavor for dairy products and beverage or esters with fruit notes like heptyl butyrate, octanoate). It also finds application as chemical intermediate for plasticizers for polymers.

\section{The "C11 cut" and its derivatives... bifonctionnality and natural bacterioactivity}

They have the rare particularity of being both long and bifunctional (presence of an carboxylic acid or ester function and a terminal double bond at the other end of the chain). This specific structure makes them highly interesting molecules which can be used on their own or as chemical intermediates for the design of tailor-made products adapted to a variety of different needs.

Undecylenic acid derivatives are also widely used for their natural bioactivity and fungi resistance (C11 chain integration into phospholipid membranes). In nature, trace quantities of C11 acid are found in sweat, tears and hair fats. It is a natural fungicide, which is effective and well tolerated. It can be used in soaps, shampoos, talcum powders and other body hygiene products. Under salt form ( $\mathrm{Zn}$ or $\mathrm{Ca}$ ), it combines natural bioresistance properties as well as surfactant improving physical stability of water based products (cosmetics). It founds applications in pharmaceuticals for human skincare: preparations as athletes' foot, haircare and anti-dandruff shampoos, deodorant, beauty creams, (antiseptic) soaps or bacteriostatic emulsifier for cosmetics. Undecylenic acid derivatives range is one of the safest, ecological, most economical and highly effective natural bacteriostatic and antifungal agents.

Undecylenic acid and methyl undecylenate are used as chemical intermediates in cosmetic industry and many of their derivatives enter in personal care compositions as surfactants, like Betain C11 or Amphoram U (undecylenamido propyl betaine), undecylenic acid diethanolamid and disodium undecylenamido MEAsulfosuccinate. Compared to chemical biocides (imidazoles, tolnaftates) used in cosmetics, they are safe and natural bacteriostatic agents.

In perfumes industry, undecylenic acid and methyl undecylenate can be used as such to provide respectively fruity-rosy note and heavy citrus note (for lilac based perfumes and anti-odors) or as chemical intermediate for:

- undecenal for rose or jasmine based formulations, key component for quality perfumes (fixing agent): quality enhancer for large volume products;

- undecenol for volume/natural freshness to floral compositions (citrus, floral note), effect increasing, especially in soap formulations;

- macrocyclic musk: e.g. cyclopentadecanolid (exaltolide, pentalide, thibetolide).

\section{A bio-renewable source for polymers (figure 2)}

In the middle of the 40 's, a group of French chemists succeeded to synthesize a monomer from undecylenic acid: 11-amino-undecanoic acid. As early as 1947, they created a new polymer from renewable sources: "PA11" polyamide, as it is known to chemists, sold under the trade name of Rilsan ${ }^{\circledR}$. By coupling 11amino-undecanoic acid with other monomers, ARKEMA developed a whole range of biobased polymers combining high performance and sustainability recognized by the $\mathrm{Rnew}^{\circledR}$ suffix. Rilsan ${ }^{\circledR}$ PA11 is the only High Performance polymer $100 \%$ based on renewable resources. For nearly 60 years, Rilsan ${ }^{\circledR}$ polyamides deliver a unique compromise of several key physical \& chemical properties including chemical \& hydrocarbon resistance, low density/light weight, impact strength, burst strength, abrasion \& scratch resistance, flexibility, electrical resistance, high temperature service and longterm ageing. Rilsan ${ }^{\circledR}$ PA 11 resins have earned a preferred material status in the most demanding applications due largely to their excellent balance of thermal, physical, chemical and mechanical properties resulting in an outstanding cost performance ratio. Processing ease is another major benefit of Rilsan ${ }^{\circledR}$ polyamide resins: injection molding, extrusion (fibers, web, tubes, multilayers, laminating, blown film, blow molding...), or rotomolding. For all these reasons, Rilsan ${ }^{\circledR}$ PA 11 has been selected by engineers for markets and applications as diverse as automotive fuel lines, pneumatic airbrake tubing, electrical anti-termite cable sheathing, oil \& gas flexible pipes \& control fluid umbilicals, sports shoes, rotomolded fuel tanks, textile, etc ...

Rilsan ${ }^{\circledR}$ PA11 can be supplied in pellets but also in powder form. Rilsan ${ }^{\circledR}$ Fine Powders are unique thermoplastic Polyamide 11 powders, developed for metal coating more than 40 years ago, that combine corrosion, chemical, abrasion cavitation erosion and impact resistance, low coefficient of friction, aesthetic surface finish (warm-to-the-touch), compatibility with food products and good hygienic properties. Rilsan ${ }^{\circledR}$ coating is a proven surface protection bringing its excellent chemical and mechanical properties.

The Rilsan Fine Powder ${ }^{\circledR}$ brand has become the global benchmark in several industries looking for the ultimate metal protection in the automotive industry, for spline shafts, sliding door, seat rails, and springs coating, and in the fluids industry, for pipes, fittings, pumps, valves coating. Other major applications include coating of dishwasher baskets, printing rollers, small parts like clips and adjustors for the textile industry, clips, springs, hinges for the building 


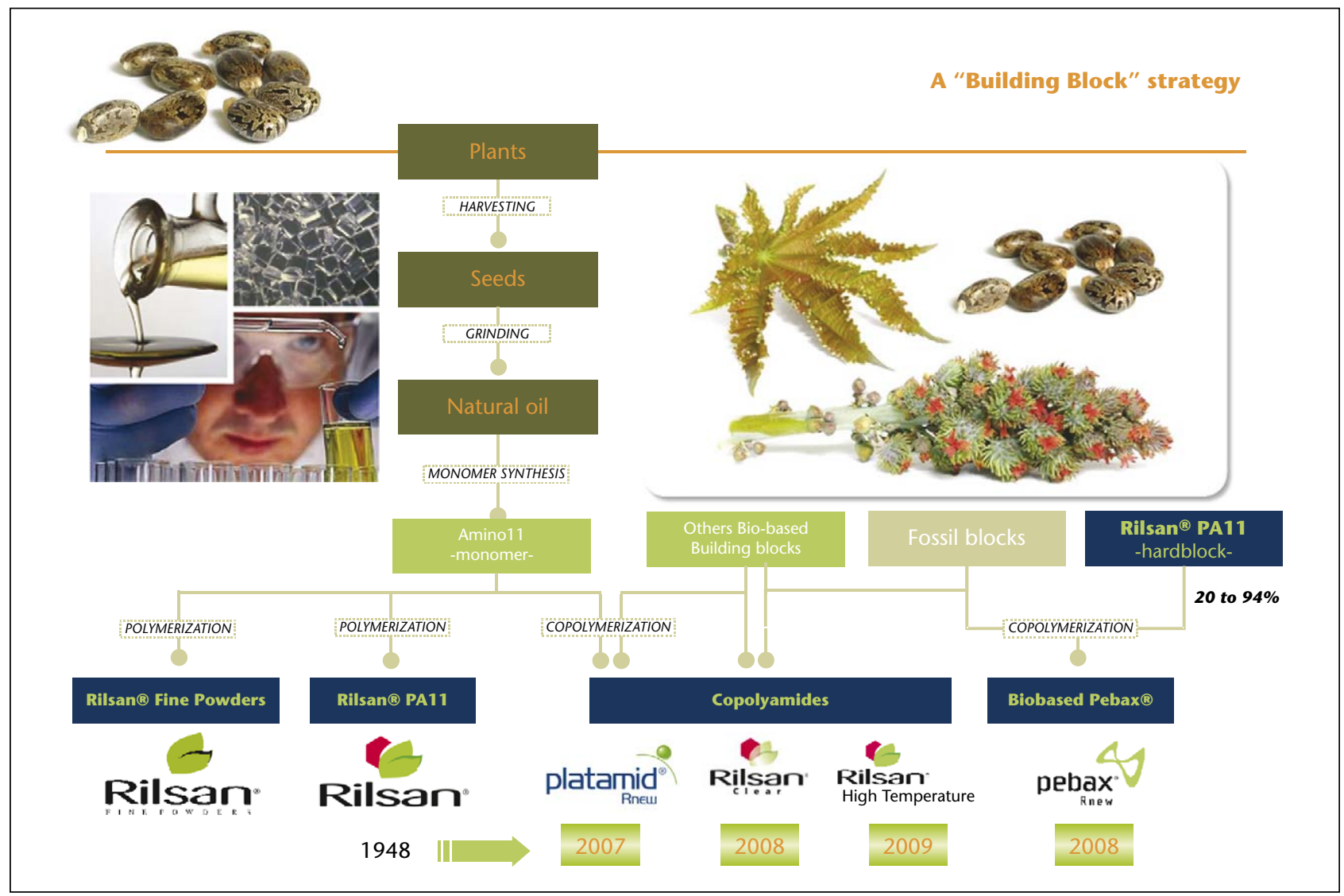

Figure 2. Image-Castor Oil Oleochemistry.

industry. Beside coating applications, Rilsan ${ }^{\circledR}$ Fine Powders can also be used as texturing additives in coil coatings and paints, as well as in Laser Sintering process.

Pebax Rnew ${ }^{\circledR}$ are plasticizer-free thermoplastic elastomers belonging to the engineering polymer family. Arkema has developed the first engineering thermoplastic elastomer range produced from renewable resources (20 to $94 \%$ of renewable organic carbon). Thanks to their unique chemistry (polyEther Block Amide - PEBA), by varying the monomeric block types and ratios, it is possible to achieve a wide range of physical and mechanical properties such as lightness, flexibility, dynamic properties due to low hysteresis (alternative flexure), energy return, resilience, outstanding properties at low temperature (impact resistance, low rigification), good resistance to most chemicals, etc. Some grades of Pebax ${ }^{\circledR}$ also bring antistatic properties, the ability to be impregnated with fragrances, soft-touch, etc.

They are easy to process by injection molding and profile or film extrusion, and can be easily melt blended with other polymers. Pebax ${ }^{\circledR}$ is suitable for many applications in markets such as sports, medical, packaging and industrial applications.

\section{Castor Oil... a vegetal refinery}

As a conclusion, every last drop is put to good use, often replacing petrochemical-based products. Castor Oil derivatives is actually a vegetal refinery! 